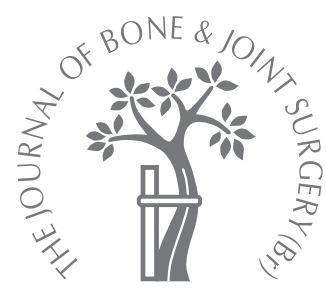

\title{
The effects of operative delay on the relief of neuropathic pain after injury to the brachial plexus
}

\author{
A REVIEW OF 148 CASES
}

\begin{abstract}
N. Kato,
M. Htut,

M. Taggart,

T. Carlstedt,

R. Birch
\end{abstract}

From The Royal

National

Orthopaedic

Hospital, Stanmore, England

\footnotetext{
N. Kato, MD, Orthopaedic Surgeon

Department of Orthopaedic Surgery

National Defense Medical

College, Tokorozawa, Saitama

359-8513, Japan.

M. Htut, MRCP,

Neurophysician

M. Taggart, RGN, RSCN,

Research Co-ordinator

- T. Carlstedt, MD, PhD, FRCS,

Professor

R. Birch, MChir, FRCS,

Professor

Peripheral Nerve Injury Unit Royal National Orthopaedic Hospital, Brockley Hill,

Stanmore, Middlesex HA7 4LP,

UK.
}

Correspondence should be sent to Professor R. Birch; e-mail: Benita.patel@rnoh.nhs.uk

(C)2006 British Editorial Society of Bone and Joint Surgery doi:10.1302/0301-620X.88B6. $16995 \$ 2.00$

$J$ Bone Joint Surg $[\mathrm{Br}]$ 2006;88-B:756-9.

Received 22 July 2005,

Accepted after revision

20 January 2006

\begin{abstract}
We investigated the effect of delay before nerve repair on neuropathic pain after injury to the brachial plexus. We studied 148 patients, 85 prospectively and 63 retrospectively. The mean number of avulsed spinal nerves was 3.2 (1 to 5 ). Pain was measured by a linear visual analogue scale and by the peripheral nerve injury scale. Early repair was more effective than delayed repair in the relief from pain and there was a strong correlation between functional recovery and relief from pain.
\end{abstract}

Pre-ganglionic injury of the adult brachial plexus provokes a characteristic constant crushing or burning pain, which is felt in the insensible hand and severe pain which shoots into the dermatome of the injured spinal nerve. Severe pain is less frequent after post-ganglionic rupture. Relief from pain by successful nerve repair has been reported ${ }^{1,2}$ and is related to re-innervation of muscle. ${ }^{3}$ Our study was the first attempt to establish whether delay before repair influenced relief from pain.

\section{Patients and Methods}

We identified two groups of patients in whom at least one spinal nerve had suffered a preganglionic injury. In the adult, the dorsal root ganglia are usually displaced into the posterior triangle of the neck. Intradural injury to the spinal nerve, without displacement of the dorsal root ganglia, is infrequent. Such an injury is determined by CT with contrast or MRI, as well as the absence of central conduction when the spinal nerve is stimulated at operation. The diagnosis is confirmed by failure of recovery and persistence of pain relating to that nerve.

Between 1986 and 1991, 85 patients were studied prospectively from before their nerve repair and throughout the process of rehabilitation. There was also a retrospective study group of 63 patients, 45 of whom had undergone a repair between 1999 and 2001 and 18 who had been admitted to our rehabilitation unit some time after their repair (1 to 11 years). All 63 patients had regularly attended our clinic, when records of recovery, pain, return to work and retraining were made. During this period there were 25 patients who had undergone nerve repair, for whom pain was absent or did not disturb sleep and daily life. They were excluded from this study, as were a further 20 patients whose post-operative attendance was irregular.

Thus a total of 148 patients with at least one avulsion was included in our study. There were 136 males and 12 females with a mean age at injury of 24.9 years (13 to 55). The causes of injury were motor-cycle accidents (121 patients), other road-traffic accidents (15) and other causes (12). The patients were followed up for a mean of 8.0 years (3 to 16 ). The dominant limb had been injured in 81 patients. Nerves were repaired either by grafting, by transfer of post-ganglionic ruptures to avulsed spinal nerves, or by extraplexal transfer using the spinal accessory, intercostal and other nerves. Reimplantation of nerve roots into the spinal cord was performed in four patients with a complete avulsion. ${ }^{4}$ The mean delay before exploration was 111.0 days (0 to 1329 ; median 63.5). The patients were divided into four groups based upon the interval between injury and exploration: group 1 (early), within one month of injury $(\mathrm{n}=61)$; group 2 (delayed), one to three months after injury ( $\mathrm{n}=$ 29); group 3 (late), three to six months after injury $(\mathrm{n}=32)$; and group 4 (neglected), more than six months after injury $(\mathrm{n}=26)$.

For each of the four groups, the severity of nerve injury and of pain was analysed by the number of nerve-root avulsions.

Assessment of pain. An individual assessment of each patient's neuropathic pain was performed using a linear visual analogue scale (VAS), scores being from zero (no pain) to ten (worst pain). Patients were asked to mark the appropriate score on a horizontal axis in order 
to quantify their pain. A worst VAS (VAS-worst) was recorded at every review, the VAS at the final review being the VAS-final. The peripheral nerve injury scale classifies neuropathic pain based upon the effect on the activities of daily living. ${ }^{5,6}$ We asked patients to describe the extent of disturbance to their work, study, hobbies and sleep and then classified the pain into four grades as follows: severe, constant disturbance of daily life, work, study and sleep (4 points); significant, able to sleep but not to work, study or enjoy hobbies (3 points); moderate, able to work, but the pain sometimes so severe as to require time off ( 2 points); and mild, aware of pain but able to lead a normal life (1 point). The peripheral nerve injury scale at its worst and the final score taken at the last review, were used in the analysis and measured at intervals from three to 16 years.

Functional assessment. The recovery for each repaired nerve was determined by serial clinical and neurophysiological examinations for all patients and by quantitative measurement of cutaneous sensibility and of post-cholinergic sympathetic function in 25 patients. The function of each joint was graded by the system of Narakas. ${ }^{4}$

The recovery for each repaired nerve element was graded as follows: good, restoration of functional active movement in at least one axis of a joint; fair, nerve regeneration proven by clinical and neurophysiological examination, but of little functional worth and poor, no regeneration. More than one element was repaired in most cases. The result for each patient was graded by the best outcome for any individual element and this was correlated with the pain scores.

Data were collected about the onset, nature and location of pain, response to drugs, response to physical method of treatment and the patient's own recognition of factors which eased or exacerbated their pain. We also included information about associated injury, time away from work or study, retraining for other occupations, operations for reconstruction, and the use of orthoses.

Statistical analysis. All values were expressed as the mean and SD. The results from the VAS were analysed by a oneway analysis of variance (ANOVA) and those from the peripheral nerve injury scores by a non-parametric test (Kruskal-Wallis) followed by the Scheffe post-hoc test. A p value of $<0.05$ was considered to be significant.

\section{Results}

The onset of pain was immediate in 59 patients, within 24 hours in 21 , within two weeks in 35 , within six weeks in 18 , within three months in ten, within six months in three and after six months in two. A total of 15 patients were intubated and ventilated for up to three weeks after injury because of associated injuries to their head, chest and spine. There were two patterns of pain: 1) constant pain in the insensible hand, usually described as crushing, burning and bursting; and 2) shooting pain always felt in the dermatome of the injured spinal nerve, but only occurring when there was a constant feature to the pain. The most important mitigating factor was distraction, either by work, study, hob- bies or conversation. Other helpful factors included warm weather and warm water. Pain was exacerbated by cold weather, cold water, stress and concurrent illness. The consumption of alcohol sometimes helped and sometimes increased pain. There were 108 patients who took analgesic medication regularly for more than six months. These provided good relief in 16 patients, some relief in 55, but were of no benefit in 37. Cannabis was helpful in 30 patients. Transcutaneous nerve stimulation was performed on 80 patients and relieved pain to some extent in 22 of those. The remaining 68 patients did not have an opportunity to undergo this treatment or refused it. Those who responded to medication were more likely to find transcutaneous nerve stimulation effective.

Patterns of nerve injury. There were three common patterns of nerve injury: total-root injury with, at least, avulsion of the lower root $(\mathrm{n}=79)$; upper-root injury with recovery in the lower root $(n=47)$; and lower-root injury with recovery in the upper root $(n=13)$. We were unable to categorise the remaining nine patients. We correlated the differences in the VAS-worst score with the type of injury. The mean VAS-worst points of the total-root, upper-root and lower-root injury groups were 9.2 (SD 0.4), 8.3 (SD 0.5) and 9.1 (SD 0.9), respectively. Lower-root injury led to a higher VAS-worst score than upper-root injury.

Severity of nerve injury and pain. The mean VAS-worst scores for patients with one to five avulsions were 7.1 (SD 0.7), 7.6 (SD 0.4), 8.6 (SD 0.3), 9.0 (SD 0.2) and 9.0 (SD 0.2), respectively. The relationship between the severity of nerve injury and pain reached statistical significance for between one and three avulsions $(\mathrm{p}<0.01)$, and between two and three avulsions $(\mathrm{p}<0.05)$.

Severity of nerve injury and delay. The mean number of avulsed spinal nerves was 3.3 (SD 0.2) in group 1, 3.3 (SD 0.2 ) in group 2, 3.0 (SD 0.2) in group 3 and 3.2 (SD 0.3) in group 4 .

Pain and delay. The median VAS-worst score was $9.0(3.0$ to 10.0$)$ in all groups. The median VAS-final was 2.0 (0 to $10)$ in group $1,3.0$ (0 to 8$)$ in group $2,4.0$ (0 to 10$)$ in group 3, and 6.0 (0 to 10 ) in group 4 . The mean VAS-worst was 8.2 (SD 0.3) in group 1, 9.1 (SD 0.2) in group 2, 8.5 (SD 0.3 ) in group 3 and 9.0 (SD 0.3 ) in group 4. The mean VASfinal was 2.6 (SD 0.3) in group $1,3.7$ (SD 0.4) in group 2, 4.0 (SD 0.5 ) in group 3 and 5.3 (SD 0.6 ) in group 4. The mean improvement in the peripheral nerve injury scale was 2.2 (SD 0.1) in group 1, 1.8 (SD 0.2) in group 2, 1.3 (SD 0.2) in group 3 and 1.1 (SD 0.2) in group 4. A shorter time interval between injury and nerve repair led to a greater improvement in neuropathic pain. The changes were statistically significant (group 1 and group 3, p < 0.01; group 1 and group 4, p $<0.01$; group 2 and group $3, \mathrm{p}<0.05)$.

Change in visual analogue and peripheral nerve injury scores over time. Pain reached maximum intensity by three months after injury in $69(81.2 \%)$ of the 85 patients studied prospectively. The mean interval to reach maximum pain intensity was 2.5 months ( 75 days; 1 to 183 ) for both scoring systems. 
Functional assessment. We repaired 284 spinal nerves in 139 patients and undertook no repair in 11. Functional recovery was good in 90 nerves $(31.7 \%)$, fair in 140 $(49.3 \%)$ and poor in $54(19.0 \%)$. The outcome for the 139 patients who had at least one nerve repair was good in 58 patients $(41.7 \%)$, fair in $58(41.7 \%)$ and poor in 23 $(16.6 \%)$.

Functional recovery by delay. In group 1, $34(56.7 \%)$ had a good result, $15(25.0 \%)$ a fair and $11(18.3 \%)$ a poor result. In group 2 the values were 11 (39.3\%), 11 (39.3\%) and six $(21.4 \%)$ respectively, in group 3 they were seven $(25.9 \%), 13(48.1 \%)$ and seven $(25.9 \%)$ and in group 4 , three $(13.6 \%), 11(50.0 \%)$ and eight $(36.4 \%)$, respectively. Functional recovery and pain. The mean VAS-worst scores for those patients with a good, fair or poor functional outcome was 8.3 (SD 0.2), 8.8 (SD 0.2 ) and 8.6 (SD 0.3) respectively and the mean VAS-final scores were 2.2 (SD 0.3 ), 3.7 (SD 0.3) and 5.0 (SD 0.5), respectively. These differences were significant (good and fair, $\mathrm{p}<0.01$; good and poor, $\mathrm{p}<0.01$; fair and poor, $\mathrm{p}<0.05)$. The mean improvement in the peripheral nerve injury scale was 2.2 (SD 0.1 ) for good, 1.9 (SD 0.1) for fair and 1.2 (SD 0.2) for poor functional outcome. These differences were also significant. Return to work. There were 131 patients who were employed at the start of the study, $78(59.5 \%)$ returned to work during the course of our study, while $53(40.5 \%)$ did not. The mean VAS-final score for those who returned to work was 3.5 (SD 0.6) and for those who didn't was 4.3 (SD 0.8).

\section{Discussion}

Pre-ganglionic lesions of the spinal nerves of the brachial plexus usually produce a highly characteristic neuropathic pain which can persist for many years. Pain is one of the most important obstacles in rehabilitation. Our study was the first attempt to analyse the correlation between the delay before nerve repair and the improvement of neuropathic pain after injury to the brachial plexus.

Our major finding was that a shorter interval between injury and repair led to a greater improvement of pain. We applied two scales in order to analyse pain. The advantage of the VAS was simplicity, but its major disadvantage was that it did not reflect the effect of pain on daily activities. The peripheral nerve injury scale attempted to fill this gap. For our study, both systems showed the same trend in the improvement of pain.

Our study also found a trend indicating that more avulsions led to a higher score for VAS-worst. Previous reports have shown that the pain was related to the number of preganglionic lesions. ${ }^{1,6,7}$ VAS-worst was related to the pattern of nerve injury. This pattern was similar to that seen in our earlier report ${ }^{4}$ of 300 consecutive patients operated on between 1989 and 1993, in whom approximately half of the lesions were incomplete and complete avulsion occurred in 52. Of these, 117 patients had damage to the upper nerves, but with recovery in the lower nerves, 23 showed injury to $\mathrm{C} 6, \mathrm{C} 7$ and $\mathrm{C} 8$, with recovery in $\mathrm{C} 5$ and
T1, and 13 had recovery for C5 and C6 with damage for C7, C8 and T1. ${ }^{4}$

Narakas $^{1}$ showed that repair of the brachial plexus relieved the pain of ruptures in $90 \%$ of his cases. Of patients with avulsions, $17 \%$ continued with disturbing pain from neurotisation for three years or more, compared with $25 \%$ of those who had not undergone surgery. Berman et $\mathrm{al}^{3}$ found a close relationship between the relief from pain and return of muscle power. The correlation between relief, measured by the McGill system, ${ }^{3}$ and the return of function was highly significant. The decrease in pain appeared to accompany, or slightly precede, the first sign of functional recovery. This reduction in pain was probably associated with returning function of the large myelinated fibres. Our study found that a shorter delay before operation led to a better functional outcome and that this gave a greater improvement in pain.

The patient's description of the pain following a closed traction lesion of the brachial plexus in Frazier and Skillern's ${ }^{8}$ study is typical: "The pain is continuous, it does not stop a minute either day or night. It is either burning or compressing. In addition, there is, every few minutes, a jerking sensation similar to that obtained by touching a Leyden jar. It is like a zig-zag made in the sky by a stroke of lightening". The constant element of this pain probably arises from disinhibition of neurones within the laminae I and II of the dorsal horn, which fire spontaneously. The convulsive pain, which may respond to anticonvulsants such as phenytoin or carbamazepine, arises from sudden outbursts of ectopic electrical activity in mechanoreceptor neurones in the damaged dorsal horn. ${ }^{9}$ Woolf, Shortland and Coggeshall ${ }^{10}$ provided an explanation for peripheral neuropathic pain by demonstrating the collateral sprouting of large, myelinated afferent fibres around nociceptor neurones in lamina II of the dorsal horn. Michaelis, Liu and Janig, ${ }^{11}$ in a rat experimental model, produced spontaneous activity in afferent fibres from skeletal muscles. Nerves to the gastrocnemius were sectioned, which induced bursting, spontaneous discharges from the neurones of the dorsal root ganglion. The conduction velocity ranged from 5 to $30 \mathrm{~m} / \mathrm{s}$, incompatible with C-fibre activity. It is possible that similar events occur within the dorsal horn after pre-ganglionic injury and that restoration of impulses from the periphery by afferent fibres from muscle underlies the relief from pain associated with the recovery of muscle function.

However, late intercostal nerve transfer is sometimes effective for the relief from pain, even when there is no realistic prospect of functional recovery of skeletal muscle. ${ }^{5}$ There are several possible explanations for these results. First, the transfer of healthy intercostal nerves into the trunk nerves of the upper limb may inhibit abnormal electrical activity within the substantia gelatinosa. Secondly, relief from pain may purely be a non-specific effect of operation, depending on the anaesthetic used, the degree of post-operative pain and the use of analgesics, or on suggestion alone. Thirdly, relief may be produced by sectioning of 
functioning axons of the posterior root system, impulses from which have in some way been reaching the central nervous system.

Other possible mechanisms may be considered. Distraction, by appropriate rehabilitation which is designed to return the patient to work and recreation as soon as possible, is particularly effective. Early surgical intervention leads to early rehabilitation because the diagnosis is established and because repairs may improve the prognosis. A few of our patients showed a dramatic improvement in their neuropathic pain immediately after operation; others showed relief from pain shortly after their return to work, suggesting that psychological factors are important. We found that the mean VAS-final of patients who returned to work ( 78 of $131 ; 59.5 \%$ ) was lower than for those who did not.

We were dismayed to find that only 61 of the 148 patients $(41 \%)$ underwent surgery within one month of injury. In an earlier study ${ }^{4}$ of 147 repairs performed between 1991 and 1993, 63 patients (43\%) underwent surgery within 14 days of injury and 22 more $(15 \%)$ within one month. The proportion of urgent repairs was even higher in 65 patients with complete lesions who had repair by vascularised ulnar nerve graft between 1975 and $1984 .{ }^{12}$ In these two series ${ }^{12}$ elements of hand function were restored in five adults by repair of C7, C8 and T1. All received surgery within 14 days.

In conclusion, we disapprove of the apparent trend towards delay in the operative treatment of these patients.
Early exploration and repair are critical in the treatment of neuropathic pain and functional impairment after injury to the brachial plexus.

No benefits in any form have been received or will be received from a commercial party related directly or indirectly to the subject of this article.

\section{References}

1. Narakas AO. The effects of pain of reconstructive neurosurgery in 160 patients with traction and/or crush injury to the brachial plexus. In: Siefried J, Zimmerman M, eds. Phantom and stump pain. Berlin: Springer-Verlag, 1981:126-47.

2. Bruxelle J, Travers V, Thiebaut JB. Occurrence and treatment of pain after brachial plexus injury. Clin Orthop 1988;237:87-95.

3. Berman J, Birch R, Anand P. Pain following human brachial plexus injury with spinal cord root avulsion and the effect of surgery. Pain 1998;75:199-207.

4. Birch R, Bonney G. Traumatic lesions of the brachial plexus. In: Birch R, Bonney G, Wynn Parry CB, eds. Surgical disorders of the peripheral nerves. Edinburgh: Churchil Livingstone, 1998:157-207.

5. Berman J, Anand P, Chen I, Taggart M, Birch R. Pain relief from preganglionic injury to the brachial plexus by late intercostal nerve transfer. J Bone Joint Surg $[\mathrm{Br}]$ 1996;78-B:759-60.

6. Birch R, Bonney G. Pain. In: Birch R, Bonney G, Wynn Parry CB, eds. Surgical disorders of the peripheral nerves. Edinburgh: Churchill Livingstone, 1998:373-404.

7. Wynn Parry CB. Pain in avulsion lesions of the brachial plexus. Pain 1980;9:41-53.

8. Frazier CH, Skillern PG. Supraclavicular subcutaneous lesions of the brachial plexus not associated with skeletal injuries: with the report of a case of avulsion of the anterior and posterior spinal roots. J Am Med Assoc 1911;57:1957-63.

9. Loeser JD, Ward AA Jr, White LE Jr. Chronic deafferentation of human spinal cord neurons. J Neurosurg 1968;29:48-50.

10. Woolf CJ, Shortland P, Coggeshall RE. Peripheral nerve injury triggers central sprouting of myelinated afferents. Nature 1992;355:75-8.

11. Michaelis $\mathbf{M}$, Liu $\mathbf{X}$, Janig W. Axotomized and intact muscle afferents but no skin afferents develop ongoing discharges of dorsal root ganglion origin after peripheral nerve lesion. J Neurosci 2000;20:2742-8.

12. Birch R, Dunkerton M, Bonney G, Jamieson AM. Experience with the free vascularized ulnar nerve graft in repair of supraclavicular lesion of the brachial plexus. Clin Orthop 1988;237:96-104. 